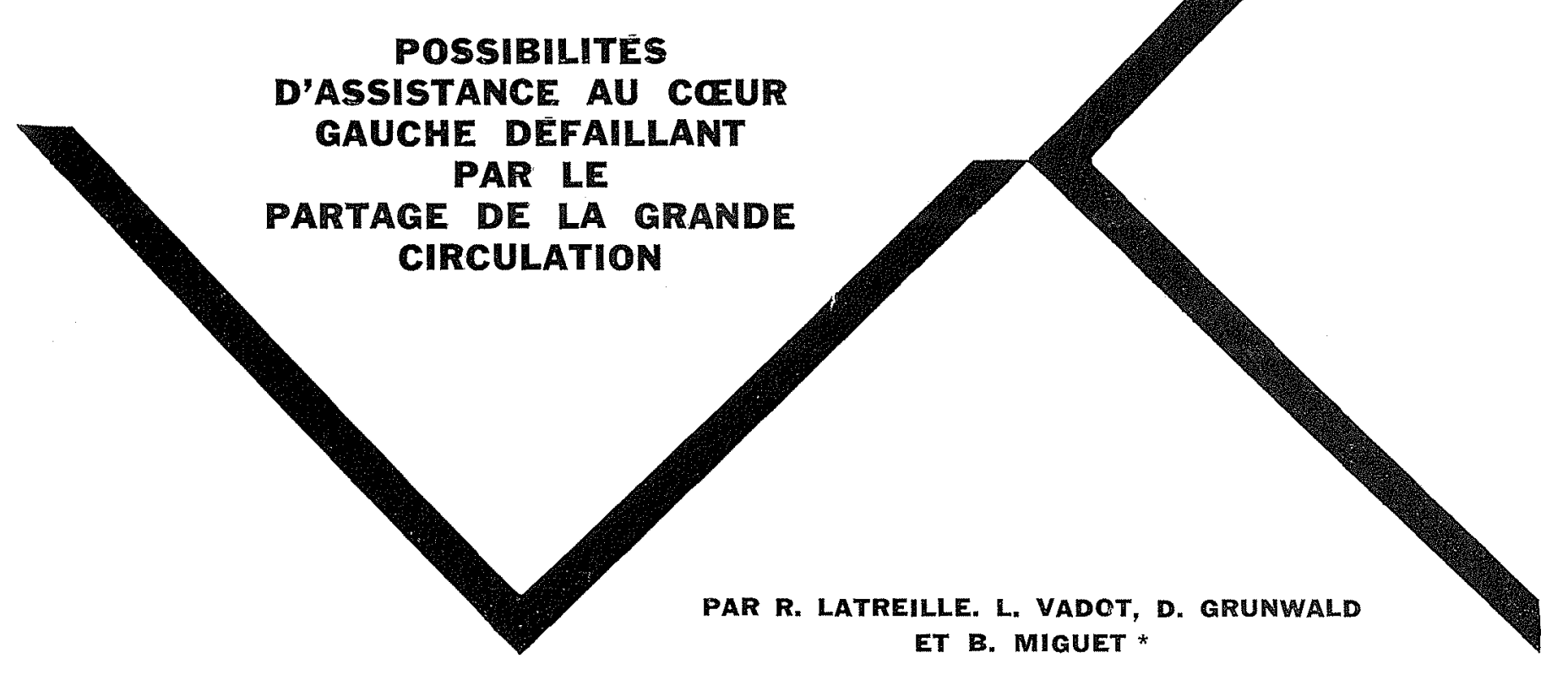

L'assistance mécanique au coeur défaillant est obtenue par tout système capable de déterminer une réduction temporaire de l'énergie que développe normalement le cour pour assurer la grande circulation. Autrement dit, pour arriver au but recherché, il importe de diminuer le travail du ventricule gauche, travail qui est fonction du débit systémique et des pressions ventriculaires et systoliques moyennes. Deux solutions de principe s'imposent donc en première analyse : soit l'abaissement de la pression aortique, c'est-à-dire des résistances péri. phériques, sans toutefois faire descendre la pression diastolique au-dessous du seuil exigé par la circulation coronaire: les procédés de contrepulsation satisfont avec élégance à ces nécessités à condition que l'ondée post-systolique artificielle soit d'un volume suffisant, injectée le plus près possible du cour et surtout exactement et uniquement pendant la diastole (ce qui pose de délicats problèmes de synchronisation avec un cour en forte tachycardie) et que d'autre part le procédé ne provoque pas une hémolyse trop prononcée sur le sang anime de violents mouvements de brassage; soit la réduction du débit ventriculaire dont une part aussi grande que possible est prise en charge par un court-circuit partiel du cœur; toutefois, cette solution présente deux inconvénients majeurs : si la circulation extra-corporelle doit assurer l'oxygénation du sang, il n'est pas possible d'en attendre un scrvice prolongé au-delà de quelques heures à cause des limites qui sont jusqu'ici inhérentes aux oxygenateurs artificiels; de là vient l'intérêt des courtcircuits du cœur gauche qui prélevent le sang

\footnotetext{
* Travail du Laboratoire de Chirurgie Expérimentale de la Faculté de Médecine de Grenoble.
}

oxygéné dans l'oreillette gauche (Carlens). Mais un autre risque persiste, commun à tous les procédés d'assistance par C.E.C., celui que représente la mise en compétition du système d'assistance el de l'activité restante du cour défaillant; ainsi, tant que le courl-circuit est partiel, le cour n'est pas soulagé.

C'est pour pallier cet inconvénient fondamental qu'un expérimentateur américain, Kuhn, a proposé en 1960 de compartimenter la grande circulation pour donner une plus grande efficacité au C.E.C. d'assistance cardiaque. Des expériences fort instructives ont montré, semble-t-il, qu'une occlusion de l'aorle abdominale associée à un court-circuit veino-artériel permettait de diminuer le travail du coeur dans la proportion de 3 à 1 par diminution du débit du ventricule gauche, en mème temps que la pression de l'aorte proximale et la circulation coronaire étaient maintenues à un niveau au moins égal à la normale. Cependant, à notre connaissance, l'étude de ce procédé de cloisonnement vasculaire n'a pas été approfondie, peut-être parce que les insuffisances des oxygénateurs condamnaient apparemment la méthode. Or, il nous a paru très fécond de reprendre celle idée du cloisomnement de la grande circulation et d'en faire le principe même de la méthode que nous cherchons à définir, méthode qui se caracterise avant tout par le partage de la grande circulation en deux territoires, l'un proximal - près du cour - celui des coronaires et des carotides, l'autre distal ou inférieur, celui des artères rénales, de l'artère mésentérique supérieure et du trone coliaque. Le travail du cour est ici réduit, en principe, par une diminution du débit ventriculaire, alors que le régime des pressions dans le territoire proximal est maintenu dans les limites physiologiques : en effel, on laisse au cour malade 
la charge de la seule circulation coronaire et encéphalique, c'est-à-dire la responsabilité d'un lit vasculaire réduit approximativement au tiers de l'ensemble du réseau systémique. Et c'est le système d'assistance qui est chargé de la circulation dans la partie distale sous-diaphragmatique, soit dans les deux tiers restants.

En somme, l'assistance au cour gauche par lo partage de la grande circulation est faite de la juxtaposition de deux manouvres essentiellement complémentaires et qui sont d'un côté l'occlusion de l'aorte thoracique descendante immédiatement en aval de la sous-clavière gauche par un ballonnet et d'autre part et simultanément la mise en route du système d'assistance, qui assure la vascularisation dans le territoir'e d'aval. Le ballonnet crée en quelques instants les deux compartiments vasculaires; quant au circuit extra-corporel d'assistance, nous en avons expérimenté deux modèles: l'un artério-artériel, unissant l'artère axillaire en amont du ballonnet à l'artère fémorale en aval, a l'avantage de la simplicité puisqu'il n'est pas utile de prévoir un système d'oxygénation; l'autre veinoartériel, unissant la veine et l'artère fémorale, comporte nécessairement un procédé assurant l'oxygénation du sang dans le circuit. C'est en travaillant et en méditant pendant un an sur le partage de la grande circulation selon ces différentes modalités que nous sommes arrivés aux quatre conclusions générales suivantes, incomplètes assurément mais, semble-t-il, déjà solidement établies :

\section{Première conclusion :}

L'occlusion aortique par un ballonnet est un geste simple, remarquablement et très rapidement efficace sur l'ischémie myocardique, mais qui exige du cour un accroissement assez sensible quoique relatif de son travail.

La simplicité de la méthode est évidente, puisqu'il suffit de monter une sonde à ballonnet dans l'artère fémorale jusqu'au niveau de l'aorte thoracique descendante. Cette sonde comporte deux prises de pression, l'une en amont, l'autre en aval du ballonnet, qui permettent de suivre les modifications tensionnelles dans les deux territoires vasculaires séparés par le ballon lorsqu'il est gonflé.

L'efficacité sur l'ischémie myocardique est véritablement spectaculaire car, dès l'occlusion aortique, la pression d'amont, effondrée au-dessous de $70 \mathrm{~mm} \mathrm{Hg}$ au cours de nos expériences par une importante spoliation sanguine, retrouve un niveau normal en quelques secondes. Le résultat est comparable à celui que provoque la compression aortique à la sortie du diaphragme lors des grandes hémorragies abdominales ou à celui, habituellement très fugace, de l'injection de vasopresseurs au cours d'une hypotension aiguë. Ainsi, se trouve éliminé et contrôlé ce facteur de gravité essentiel qu'est l'insuffisance coronaire fonctionnelle secondaire à la chute de la pression de perfusion du myocarde; et cette amélioration remarquable de l'irrigation coronaire par le retour d'une pression aortique normale autorise probablement l'ouverture rapide d'anastomoses de suppléance autour du foyer infarci.

Cependant, les risques de la méthode ne peuvent être négligés, car ils sont indiscutables : l'élévation de la pression d'amont obtenue en quelques instants, donc assez brutalement, s'accompagne d'une relative augmentation du débit cardiaque et, par voie de conséquence, d'un accroissement de l'énergie exigée du cour. Cette sollicitation à un travail accru est toutefois relative et reste assez éloignée de l'énergie développée par le cœur dans les conditions normales si l'on retient que le cœur défaillant ne fournit plus, approximativement, avant le moment de l'occlusion aortique, que la moité du travail normal du ventricule gauche. Toutefois, elle exposerait à l'aggravation rapide de la défaillance cardiaque si elle n'était compensée dans le même temps par l'amélioration de la vascularisation myocardique. C'est dire cependant la prudence avec laquelle l'occlusion de l'aorte thoracique en aval d'un ventricule gauche défaillant doit être provoquée; c'est dire encore qu'une occlusion incomplète peut être insuffisante et mieux supportée; il se peut même que la situation hémodynamique soit rétablie sans le secours d'un systeme d'irrigation du territoire d'aval par une occlusion partielle et assez courte (de l'ordre de quelques minutes), maintenue juste le temps nécessaire pour "remeltre en selle " un cœur plus atteint par l'ischémie fonctionnelle due à l'hypotension qu'à l'infarcissement lui-même.

\section{Deuxième conclusion :}

Le court-circuit artériel entre axillaire et fémorale est insuffisant pour assurer la circulation sous-diaphragmatique en normothermie tant que le débit du shunt extérienr est faible.

Lorsque le circuit extra-corporel mis en place comporte une pompe capable de forts débits, il est possible, dès le moment où l'aorte est compartimentée, d'établir dans le shunt extérieur un débit égal au débit sous-diaphragmatique physiologique et l'égalité des pressions dans les territoires en amont. et en aval du ballon apporte la preuve que la circulation splanchnique est redevenue normale. Mais établir des pressions égales de part et d'autre de l'occlusion aortique, c'est à coup sûr perdre tout le bénéfice de cette occlusion et nous avons done cherché avec quel débit minimal le shunt extéricur peut satisfaire aux besoins vitaux du foie et des reins. En effet, s'il était possible de diminuer dans de fortes proportions cette circulation sous-diaphragmatique, le gain serait très sensible sur le plan des débits, si l'on veut bien se rappeler que le débit des artères rénales représente $23 \%$ et celui de l'artère mésentérique supérieure et du tronc coliaque $28 \%$ du débit global du cœux. Celui-ci étant approximativement fixé à $80 \mathrm{~cm}^{3} / \mathrm{kg}$. mn pour que soit maintenue en normothermie une pression aortique de $120 \mathrm{~mm} \mathrm{Hg}$, le seul débit splanchique est dans les mêmes conditions de $40 \mathrm{~cm}^{3} / \mathrm{kg} . \mathrm{mn}$. Cherchant délibérément la vascularisation minimale dans ce territoire sous-diaphragmatique, nous avons entretenu un débit de $20 \mathrm{~cm}^{3} / \mathrm{kg}$. mn, soit moitié moindre que le débit normal en aval du ballonnet, tout en gardant environ $30 \mathrm{~cm}^{3} / \mathrm{kg} \cdot \mathrm{mn}$ pour la vascularisation d'amont, chiffre qui permet le maintien d'une pression aortique proximale à peu de chose près normale. C'est en suivant ce schéma que nous avons abouti aux conclusions suivantes :

$1^{\circ}$ Si l'on entretient une circulation d'aval avec 
un débit de $20 \mathrm{~cm}^{2} / \mathrm{kg} \cdot \operatorname{mn}$ (soit $300 \mathrm{~cm}^{3} / \mathrm{mn}$ pour un chien de $15 \mathrm{~kg}$ ), on obtient une pression de $60 \mathrm{~mm} \mathrm{Hg}$ dans l'aire splanchnique : cette pression est la moitié de la pression dans l'aorte proximale et peut être le plus souvent obtenue par un courtcircuit axillaire-fémorale simple, sans pulseur;

$2^{\circ}$ Cette circulation minimale protège contre l'ischémie pendant une heure environ et e'est ce que plusieurs auteurs américains avaient noté dès 1955 : King et Shumaker, puis Cross ont établi que s'il faut un débit de $40 \mathrm{~cm}^{3} / \mathrm{kg}$. $\mathrm{mn}$ pour maintenir une pression d'aval normale, un débit moitié moindre ( 20 et même $15 \mathrm{~cm}^{3} / \mathrm{kg} \cdot \mathrm{mn}$ ) assure une pression de $60 \mathrm{~mm} \mathrm{Hg}$ qui protège les organes sous-diaphragmatiques contre les effets de l'ischémie pendant 60 à $70 \mathrm{mn}$. Or il nous est apparu qu'en aucun cas cette circulation minimale ne saurait suffire andelà de ce court laps de temps, et à chaque fois que nous avons prolongé la circulation artificielle d'aval sous cette faible pression de $60 \mathrm{~mm} \mathrm{Hg}$, il est survenu entre la cinquième et la sixième heure un col. lapsus rapide et généralisé, mortel en quelque 8 ou 10 h. Il nous a semblé que l'occlusion de l'aorte, malgré la dérivation artério-artérielle, créait les conditions d'apparition du choc par sténose de l'artère mésentérique supérieure, tel que l'a décrit Lillehei; rappelons que les conséquences de la réduction du débit mésentérique supérieur sont la stagnation du sang dans le territoire porte compris entre deux systèmes capillaires et par voie de conséquence une insuffisance de la circulation de retour, puis une ischémie irréversible de l'intestin grêle, responsable de la nécrose de la muqueuse intestinale à partir de la cinquième ou sixième heure après le début du choc. Or c'est dans le même délai que sont apparus chez nos sujets le choc irréversible qui a mis fin aux expériences. En somme, si l'oir veut préserver le foie et les reins contre l'ischémie pendant plusieurs heures et a fortiori plusieurs jours, il est indispensable d'entretenir une circulation optimale à peine inférieure à la circulation splanchnique normale, ceci tant que la température reste à $37^{\circ} \mathrm{C}$.

\section{Troisième conclusion :}

La réfrigération de la dérivation axillainefémorale provoque l'hypothermie du territoire splanchnique en aval du ballonnet intra-aortique et autorise dans cet élage inférieur une circulation a très faible débit, sans comprometle la survie des organes sous-diaphragmatiques (sché$\operatorname{ma} \mathrm{n}^{\circ} 1$ ).

C'est pour tourner les difficultés nées du maintien d'une circulation splanchnique minimale ou soidisant telle que nous avons alors envisagé de diminuer les besoins des viscères abdominaux par abaissement de leur température. Foie, intestins et reins étant protégés par une hypothermie plus ou moins profonde, selon les principes qui seront rappelés dans l'exposé suivant, on peut se contenter alors d'assurer la circulation sous-diaphragmatique par un shunt extérieur, au débit très faible, représentant la moitié ou le $1 / 3$ du débit normal selon la profondeur du refroidissement. Cet abaissement de la température viscérale est obtenue très simplement par réfrigération du circuit extra-cor-



Coclusion le l'aorte thoricinle et hypothemie du territoire sous-diaphragmatique par rêrifúration de la dérivation axillaire-fémorale. 1/ Occhusion de l'aorte thoracique et hypothermie sous-diaphragmatique.

porel et nous avons ainsi pratiqué dix expériences selon un protocole opératoire qui associait l'obligatoire occlusion aortique et une dérivation axillairefémorale sur laquelle était placée un échangeur thermique; dès l'occlusion de l'aorte, la pression inférieure s'effondre comme il est habituel et se stabilise en moyenne à la moitié de la pression d'amont $(60 \mathrm{~mm} \mathrm{Hg}$ contre $120 \mathrm{~mm} \mathrm{Hg})$; puis la réfrigération du sang dans le circuit extra-corporel abaisse la température à $25^{\circ} \mathrm{C}$ dans la veine cave inférieure en dix minutes environ; cependant, cette hypothermie ne reste pas limitée à l'étage inférieur et par le retour cave inférieur elle s'étend peu à peu au cour et à l'encéphale, quoiqu'il existe toujours un sensible décalage entre la température de l'étage sous-diaphragmatique et celle du cour et qui se chiffre en moyenne à 3 ou $4^{\circ} \mathrm{C}$ d'écart, dépendant d'ailleurs pour une bonne part de l'importance du débit dans le shunt. Cette généralisation progressive de l'hypothermie qui gagne l'étage supérieur, en amont du ballonnet aortique, est un grave inconvénient, car elle déclenche inévitablement l'apparition de frissons; or une anesthésie générale, qui pourrait prévenir cette réaction au froid, est exclue si l'hypothermie doit être maintenue plusieurs dizaines d'heures. C'est pourquoi à la réfrigération du shunt extérieur il paraît indispensable d'ajouter un système de réchauffement - soit, pour le sang veineux qui revient au cour, grâce à une sonde chauffante placée dans la veine cave inférieure à l'embouchure des veines sus-hépatiques - soit, pour l'encéphale, par réchauffement de surface de la tête. Ainsi les centres thermo-régulateurs de l'encéphale échappant aux variations de température, les risques de frissons peuvent être pratiquement supprimés; c'est du moins ce que nous pensons préciser lors de prochaines expériences. 


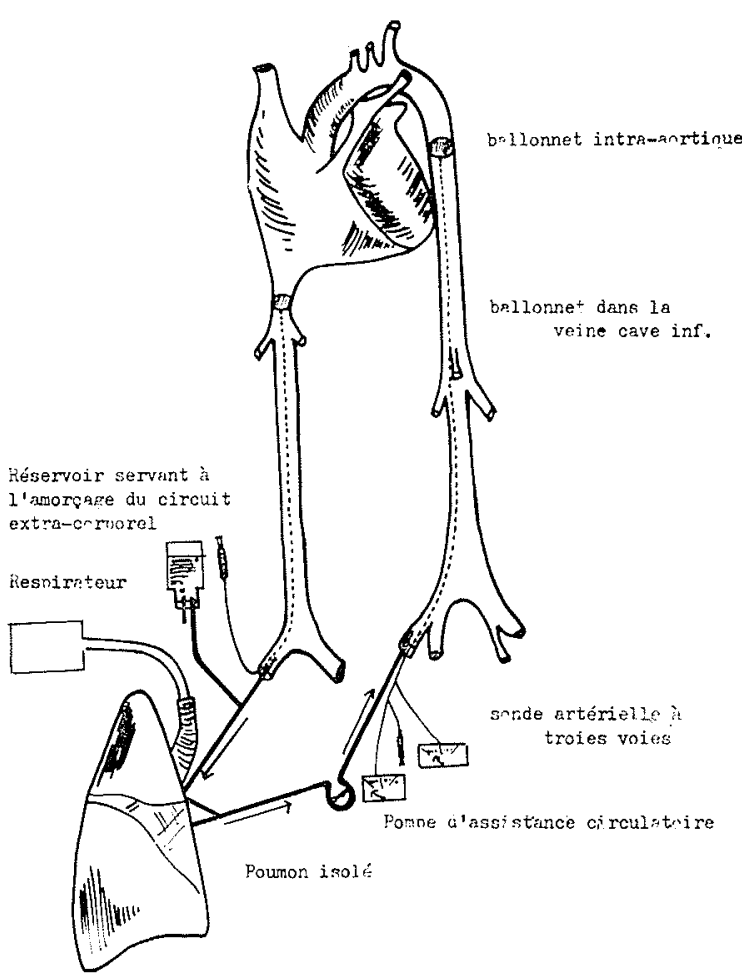

Double cloignne-"ent de $1:$ grance circulation et court-circlit entre la veine et l'artère fémorales comortunt une oxygénation par un pouson 1 solé

2/ Cloisonnement de la grande circulation et oxygénation par poumon isolé.

\section{Quatrième conclusion :}

Le circuit d'assistance circulatoire établi entre une veine et une artère fémorale assure la vascularisation autonome du territoire sous-diaphragmatique en aval du ballonnet intra-aortique, à condition que l'oxygénation du sang en soit réalisée par un poumon isolé maintenu en survie artificielle (schéma $\mathrm{n}^{\circ} 2$ ).

Ce dernier aspect de la question, pour nous aussi le plus récent, tient tout entier dans les possibilités qu'apportent, à notre avis, les acquisitions nouvelles sur le maintien en survie d'organes isolés et leur utilisation dans l'assistance aux organes défaillants; nous allons voir que le poumon est de ces organes promus à un nouveau destin. En eflet, en poussant à l'extrême le principe du cloisonnement de la grande circulation, il est logique de provoquer non seulement le partage du territoire artériel, mais également celui de la circulation de retour par un ballonnet gonflé dans la veine cave inférieure juste avant son abouchement dans l'oreillette droite. Alors se trouvent séparés deux territoires vasculaires distincts, l'un supérieur dépendant de l'activité cardiaque et dont la circulation de retour se fait par la veine cave supérieure, l'autre inférieur, désormais indépendant du cour et des poumons du sujet assisté, et pour lequel il faut prévoir une circulation extra-corporelle de suppléance qui assure à la fois la mobilisation et l'oxygénation du sang. Nous avons ainsi mis au point un protocole opératoire qui se résume dans le prélèvement du sang au niveau de la veine fémorale, son passage dans un poumon isolé obtenu par pneumonectomie, et sa réinjection par une pompe dans l'artère fémorale du même côté. Cependant, par les vaisseaux fémoraux du, côté opposé, sont montées deux sondes à ballonnet, l'une artérielle qui assure l'occlusion aortique, l'autre veineuse pour le blocage de la veine cave inférieure. Cinc expériences conduites selon ces modalités nous ont conduit aux premières conclusions suivantes :

$1^{\circ}$ L'insuffisance du retour veineux est un obstacle essentiel au moment d'amorcer le circuit extracorporel et deux précautions sont à prendre au moment crucial de la mise en train du système d'assistance - d'une part, le blocage de la veine cave inférieure par un ballonnet crée une hypertension et un reflux du sang veineux vers la veine fémorale; encore faut-il éviter avec soin que le ballon ne vienne obturer une veine sus-hépatique d'autre part, et ceci nous a paru essentiel, le retour veineux est sous la dépendance de la pression artérielle dans l'aorte abdominale; or en période de choc ou dès que le ballonnet aortique est gonflé, cette pression tombe à quelques dizaines de $m m \mathrm{Hg}$ et le sang s'arrête dans le territoire splanchnique; pour amorcer le circuit extra-corporel d'assistance dans ces conditions hémodynamiques, il est donc indispensable de lancer dans l'artère fémorale une première charge liquidienne sous forte pression et qui doit représenter au minimum plusieurs centaines de $\mathrm{cm}^{3}$; le dextran anti-sludge nous parait devoir être préféré au sang et c'est au prix de cette première injection massive dans l'aire splanchnique que l'on obtient, au bout de quelques instants, un retour veineux abondant et désormais à peu près stable. Il est toutefois de la plus élémentaire prudence de prévoir l'addition répétée de liquide supplémentaire dans le circuit extra-corporel à chaque fois que le retour veineux manifeste quelques défaillances; c'est pourquoi nous disposons sur ce circuit, entre le poumon et la pompe, d'un réservoir placé à la hauteur de la veine fémorale et dont le contenu se substitue au sang veineux automatiquement lorsque la veine tend à se collaber cost-à-dire toujours au début de l'assistance et de temps en temps au cours de l'expérience.

2" L'oxygénation du sang recueilli par la veine fémorale pose à son tour un problème vital si l'on veut bien se rappeler que la circulation d'assistance peut devoir se prolonger plusieurs dizaines d'heures et qu'il n'existe pas d'oxygénateur artificiel capable d'assurer aussi longtemps une oxygénation sanguine satisfaisante sans provoquer une hémolyse bientôt incompatible avec la vie. L'utilisation d'un poumon isolé, prélevé sur un animal et placé sur le circuit extra-corporel, nous paraît apporter une solution pleine d'avenir : en effel, les deux poumons d'un chien de bonne taille sont capables d'oxygéner $40 \mathrm{~cm}^{3} / \mathrm{kg}_{\text {g }} \mathrm{mm}$, débit du shunt veino-artériel, à condition qu'ils soient maintenus dans un milieu physiologique, et ventilés correctement par les variations de la pression négative entretenue dans la cloche. La simple gravité suffit à provoquer la circulation pulmonaire si le poumon est placé à quelques centimètres au-dessous de la canule veineuse. Cette position du poumon isolé par rapport à la veine fémorale est du reste capitale; trop élevée, elle détermine un arrêt de la circulation veineuse; trop basse, même de très peu, elle provoque un wedème 
aigu du poumon et un infarcissement généralisé de l'organe isolé.

Quoi qu'il en soit des difficultés de mise au point que nous avons jusqu'ici rencontrées, cette méthode nous semble pouvoir devenir rapidement la plus complète dans le traitement de la défaillance aiguë du ventricule gauche, car le cour est alors totalement libéré de la charge que représente toute la circulation sous-diaphragmatique et, en aval du ballonnet intra-aortique, la circulation artificielle s'effectue dans des conditions hémodynamiques aussi proches que possible des conditions normales.

\section{Conclusions}

Le partage de la grande circulation permet en règle générale de soulager le cœur défaillant des $2 / 3$ environ du travail qu'il effectue au repos et en normothermie, puisque le système d'assistance prend à sa charge la circulation sous-diaphragmatique tout entière - tantôt cette circulation splanchnique s'effectue sous basse température el sous faible débit, la réfrigération de l'étage inférieur étant assurée par refroidissement d'un circuit extracorporel artério-artériel - tantôt la circulation splanchnique s'effectue sous pression normale et en normothermie, assurée par un circuit extracorporel veino-artériel qui comporte l'oxygénation du sang veineux par un poumon isolé en survie artificielle. Que la circulation sous-diaphragmatique soit maintenue par un circuit artériel réfrigérant ou par un circuit veino-artériel oxygénant, le point commun aux deux procédés reste l'occlusion aor. tique qui compartimente le lit vasculaire et ne laiss: au cour défaillant que le territoire coronaire el carotidien.

Problèmes thermiques et hémodynamiques

L'idée du partage de la grande circulation qui vient d'être exposée et les conséquences cliniques que son application entraine, conduisent à des modifications hémodynamiques importantes du réseau systémique. Nous nous proposons, dans ce qui suit, d'examiner les problèmes ainsi posés et de présenter les résultats expérimentaux obtenus au cours de cette étude.

Le but à atteindre dans tout traitement hémodynamique de l'insuffisance cardiaque est une réduction temporaire de l'énergie demandée au cour. Réduction qui doit permettre au myocarde de récupérer pour compenser sa défaillance, mais qui doit s'accommoder aussi des besoins de l'organisme. L'élément sur lequel on s'efforce de joller ici est une réduction du débil systémique. Si en effet, tout en maintenant le niveau moyen des pressions, on réduit le débit d'irrigation systémique, on aura du même coup diminué l'énergie demandée au myocarde.

Le partage au sens strict du mot de la grande circulation réalise parfaitement cette idée. Fn effet, un clampage complet de l'aorte thoracique et de la veine cave inférieure au niveau de l'oreillette permet un isolement de la circulation sous-diaphragmatique qui sera assurée extérieurement par pompe et oxygénateur, cependant que le cœur fonctionnera à énergie réduite sur le réseau susdiaphragmatique. Ce schéma ne pose pas dans l'immédiat de problème hémodynamique, mais il nécessite évidemment pompe et oxygénateur.

La réduction du débit sous-diaphragmatique sans modification au circuit naturel d'oxygénation présente un évident intérèt de simplicité. Cette réduction peut être obtenue par deux procédés ou par leur combinaison :

$1^{\circ}$ diminution des besoins par hypothermie;

$2^{\circ}$ diminution du débit jusqu'à la limite de survivance des organes, en admettant une certaine réduction d'activité fonctionnelle.

Nous allons examiner successivement les mécanismes de ces deux procédés.

\section{HYPOTHERMIE SPLANCHNIOUE.}

L'intérêt de l'hypothermie est de réduire les besoins métaboliques el par conséquent le débit sanguin, véhicule des agents métaboliques. Pour la facilité de la compréhension, on peut, sans grosse erreur, réduire les échanges a la consommation de l'oxygène. Or, on sait que, pour un organe donné, dans les conditions basales, le débit d'oxygène nécessaire est lié à la température absolue par une relation de la forme:

$$
\mathrm{Q}=\mathrm{A} \cdot e^{-\mathrm{\textrm {B }} / \mathrm{I}}
$$

$Q$ étant le débit d'oxygène, ou le débit de sang pous: une saturation donnée;

'T la température absolue;

$A$ et $B$ des constantes dépendant de l'organe et du sujet.

Pour fixer les idées, s'il faut un litre de sang par minute pour irriguer un organe à $37^{\circ} \mathrm{C}$, il ne faudra plus que $1 / 2$ litre à $30^{\circ} \mathrm{C} ; 1 / 4$ de litre à $20^{\circ} \mathrm{C}$; $1 / 10^{\circ}$ de litre à $10^{\circ} \mathrm{C}$ et 0,04 litre à $0^{\circ} \mathrm{C}$.

Le refroidissement paraît donc offrir un moyen intéressant de réduction du débit. Les difficultés toutefois vont apparaître en cherchant à le mettre en wuvre de facon simple.

Supposons, ainsi qu'il a élé indiqué précédem-

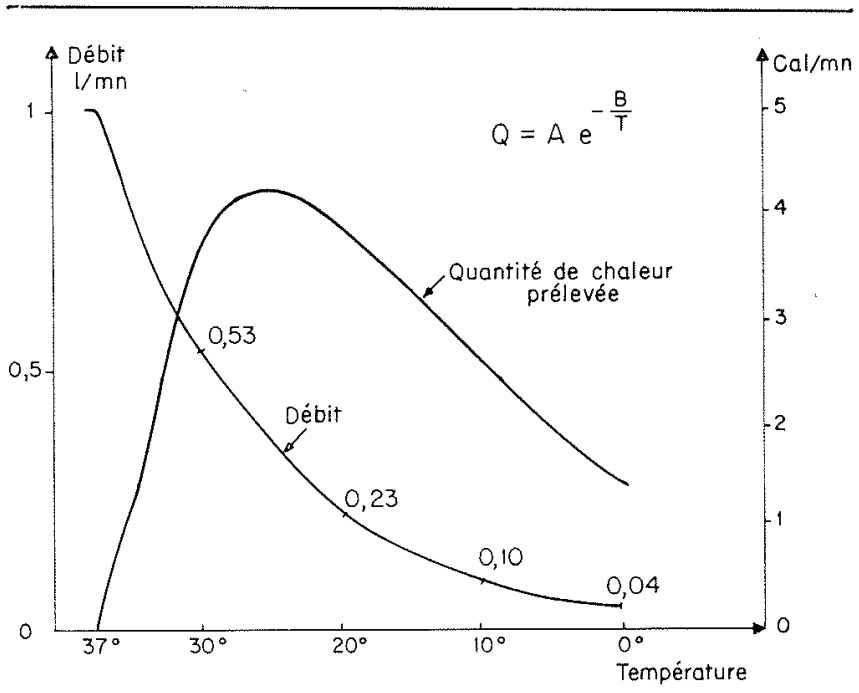

3/ Variation en fonction de la temperature, du débit d'irriration admissible et de la quantité de chaleur prélevée. 


\section{R. LATREILLE et al,}

ment, que l'on alimente l'aire splanchnique par une dérivation artério-artérielle axillaire-fémorale, après avoir clampé l'aorte thoracique. Un échangeur thermique placé sur cette dérivation assure l'abaissement de température. Il est évident que, dans de telles conditions, le retour veineux froid par la veine cave inférieure, va provoquer un abaissement général de la température. On assistera donc d'une part à un refroidissement électif accentué de l'aire splanchmique et à un refroidissement secondaire moins intense du reste de l'organisme. Comment vont se répartir les températures dans un tel système? C'est là une question à laquelle il est difficile de répondre par de simples considérations théoriques, dans l'ignorance où l'on est des perméabilités capillaires et des échanges calorifiques tissulaires des différents organes; aussi, l'expérience reste-t-elle la meilleure source d'information.

Le montage est effectué dans ce but sur le chien avec clampage aortique par sonde à ballonnet introduite par une fémorale. La dérivation axillairef'émorale comprend, outre l'échangeur thermique, une pompe volumétrique. Cette dernière n'a, bien entendu, aucun rôle d'appoint énergétique; par contre, elle permet de vaincre les résistances des canules tout en fixant exactement le débit d'irrigation froide. Pour éviter toute équivoque, disons qu'il s'agit d'une pompe doseuse.

Dans une première expérience, le débit de sang froid injecté est maintenu constant. La température d'injection, par suite de l'inertie thermique de l'échangeur, s'abaisse au cours de la perfusion de 22 à $18{ }^{\circ} \mathrm{C}$. Dans le même temps, la température du retour veineux par la veine cave inférieure s'abaisse d'abord très vite, puis plus lentement, pour atteindre $21^{\circ} \mathrm{C}$ en fin de refroidissement. La température moyenne de l'aire splanchnique peut alors être évaluée à $19,5^{\circ} \mathrm{C}$. La température au niveau du cœur baisse également, mais plus lentement et atteint $25^{\circ} \mathrm{C}$ en fin de refroidissement. Une évolution analogue avec classement inverse des températures apparaît lors du réchauffement. L'abaissement de température cardiaque s'accompagne d'une réduction de la fréquence, le rythme restant sinusal.

On constate donc par cette expérience que le refroidissement secondaire de l'organisme est important. Toutefois, on n'a pas utilisé ici une possibilité de la méthode, à savoir la réduction du débit splanchnique autorisée par le refroidissement.

Au cours d'une deuxième expérience, tout en utilisant le même montage, on réduit progressivement le débit injecté au fur et à mesure que la température splanchnique baisse. L'effet de cette réduction de débit se traduit immédiatement par un écart beaucoup plus grand entre température splanchnique et température cardiaque. Alors que précédemment, en fin de refroidissement, le cour était à $25^{\circ} \mathrm{C}$, la réduction de moitié du débit d'irrigation domne une température cardiaque au même temps de $30,5^{\circ} \mathrm{C}$, ce qui autorise une manœuvre plus longue.

Si on revient à la courbe donnant la réduction possible du débit en fonction de la température, il est facile d'en déduire la quantité de frigories injectées. On constate ainsi que cette quantité do frigories croît d'abord lorsque la température baisse jusque vers $25^{\circ} \mathrm{C}$ où un maximum est atteint; puis la quantité de frigories décroît régulièrement. Il y a donc intérêt, du point de vue thermique, si on veut appliquer le procédé de réduction de débit systémique par refroidissement splanchnique, à utiliser tout de suite des températures très basses; on réduira ainsi les effets du refroidissement secondaire (fig. 3).

Il semble néanmoins que l'application du procédé pour une durée assez grande, de l'ordre de plusieurs heures, nécessite un réchauffement compensateur partiel. Deux possibilités paraissent s'offrir pour effectuer ce réchauffement:

- soit par introduction d'un élément chauffant dans la veine cave inférieure (un échangeur de ce type a été proposé comme refroidisseur pour l'hypothermie légère);

- soit par réchauffement céphalique superficiel. Ce dernier procédé, non sanglant, autorise un bon apport de calories par suite de l'importante irrigation céphalique. (Nous avons appliqué avec succès ce mode d'échange thermique pour la conduite d'hypothermie en vue d'intervention cardiaque.)

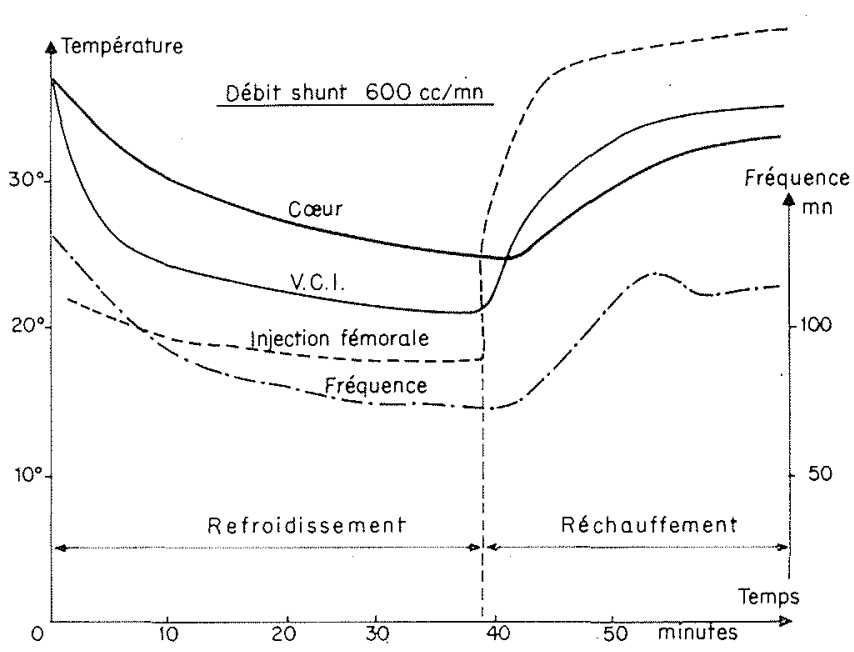

4/ Evolution des températures lors d'une hypothermie splanchnique avec débit de shunt constant.

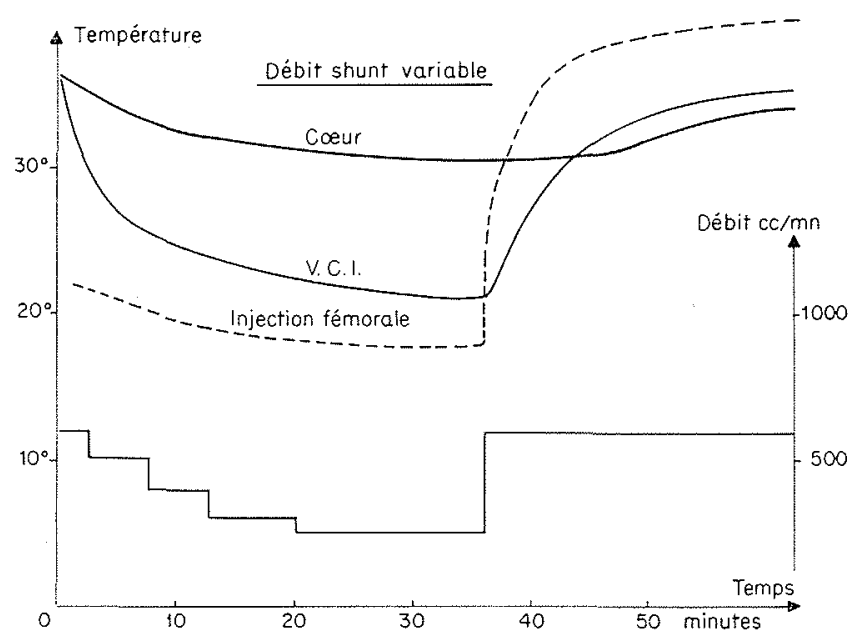

5/ Evolution des températures lors d'une hypothermie splanchnique, avec débits de shunt décroissants. 
DIMINUTION DE L'IRRIGATION SPLANCHNIQUE EN NORMOTHERMIE.

Si on convient d'admettre une certaine diminution d'activité fonctionnelle, il paraît possible de réduire notablement le débit splanchnique même en normothermie, pour autant que cette réduction soit limitée à quelques heures (voir plus haut).

On peut compter que chez l'homme, les débits se répartissent pour $1 / 3$ et $2 / 3$ entre les zones suset sous-diaphragmatiques (une répartition analogue existe chez le chien). Dans ces conditions, une réduction de moitié de l'irrigation sous-diaphragmatique entraine une réduction globale de $1 / 3$ da débit cardiaque et par suite une réduction du $1 / 3$ de l'énergie demandée au myocarde. C'est tout au moins ce qu'on peut admettre en premiere approximation.

La réalisation de la réduction du débit inférieur se fait par un montage analogue à celui utilisé pour l'hypothermie précédente, l'échangeur n'étant toutefois pas utilisé. Il est commode d'effectuer le clampage complet de l'aorte par le ballonnet de Ia sonde et de commander le débit inférieur par la
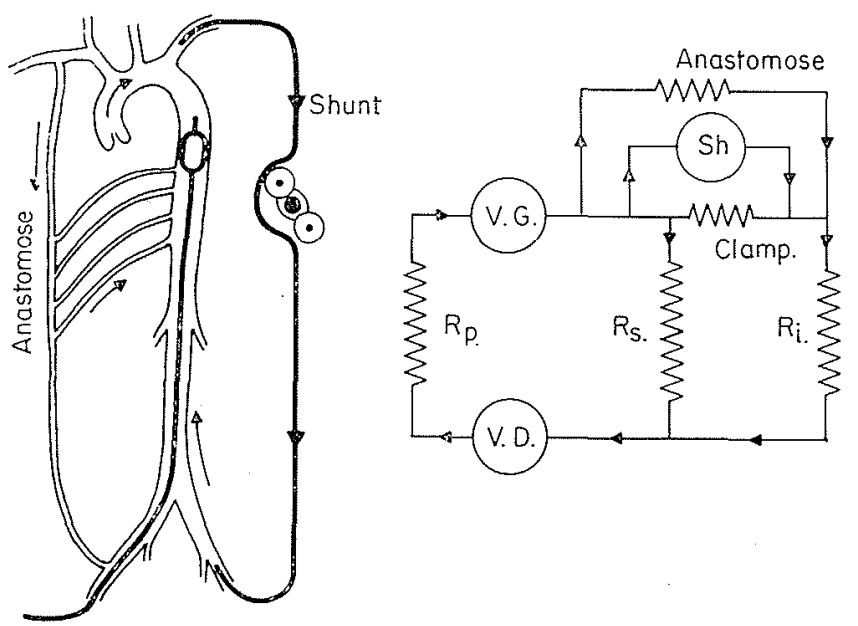

6/ Dérivations artificielle et naturelle dans le clampage de laorte, avec représentation analogique du circuit.



7/ Evolution des pressions dans les territoires inférieur el supérieur, en fonction du débit du shunt. pompe-doseuse du shunt externe. Le schéma ainsi réalisé se complique en fait par la présence d'anastomoses naturelles reliant les territoires supérieur et inférieur (circuit des artères mammaires internes et intercostales). L'existence de ce shunt naturel interdit une exclusion totale du territoire inférieur par le seul clampage au niveau de l'aorte thoracique. Ceci ne constitue d'ailleurs pas une difficulté dans notre cas, puisque l'on recherche à maintenir une certaine irrigation sous-diaphragmatique; mais il faudra en tenir compte dans l'interprétation de l'évolution des pressions.

Le montage étant réalisé, on suit l'évolution des pressions inférieure el supérieure en fonction du débit passant par le shunt extérieur. Ces pressions sont obtenues par le double cathéter joint à la sonde à ballonnet. Le clampage de l'aorte étant total et le débit du shunt extérieur étant nul (pompe doseuse arrêtée), on observe une élévation importante de la pression supérieure et un abaissement considérable de la pression inférieure. Cette dernière toutefois n'est jamais nulle par suite de l'existence du débit anastomotique.

Partant de cette situation, la mise en marche de la pompe doseuse et l'augmentation progressive du débit du shunt extérieur s'accompagnent d'une évolution opposée des pressions. La pression de l'étage supérieur s'abaisse cependant que celle de l'étaģe inférieur s'élève. La première conserve naturellement son caractère pulsatile, mais la seconde ne reflète aucun effet de rythme cardiaque; seules les systoles artificielles de la pompe doseuse apparaissent aux gros débits.

Cette évolution se poursuivant, il arrive un moment où les pressions inférieure et supérieure se rejoignent. On retrouve alors sensiblement le niveau initial de pression artérielle, et on peut admettre que, dans ce cas, le débit traversant le shunt extérieur est égal au débit normal interrompu par le clampage de l'aorte; l'aire splanchnique a retrouvé son irrigation normale.

En gros, la pression du territoire inférieur présente une variation linéaire en fonction du débit du shunt. Ce fait indique que la résistance du circuit n'est pas modifiée. Ainsi qu'il a été dit, la pression conserve une certaine valeur pour un débit nul du shunt extérieur; elle ne peut s'annuler que par une inversion du débit du shunt extérieur compensant l'effet anastomotique. L'extrapolation de la droite représentative de l'évolution des pressions permet de déterminer la valeur du débit anastomotique. Dans l'expérience, considérée, celui-ci est de $300 \mathrm{~cm}^{3} / \mathrm{mn}$ pour une pression supérieure de $150 \mathrm{~mm} \mathrm{Hg}$. Pour la pression moyenne artérielle initiale de $90 \mathrm{~mm} \mathrm{Hg}$, ce débit serait de $180 \mathrm{~cm}^{3} / \mathrm{mn}$, soit environ $25 \%$ du débit total d'irrigation inférieure. Ce chiffre montre l'importance des dérivations naturelles.

L'évolution de la pression supérieure, sensiblement linéaire au voisinage du débit normal (égalité des pressions inférieure et supérieure), se traduit par une courbe à concavité dirigée vers le bas lorsque le débit du shunt extérieur diminue. Si on convient d'admettre que la résistance du réseau supérieur n'est pas modifiée, l'évolution du débit d'irrigation supérieur est parallèle à celle des pressions. L'augmentation de la pression supérieure 


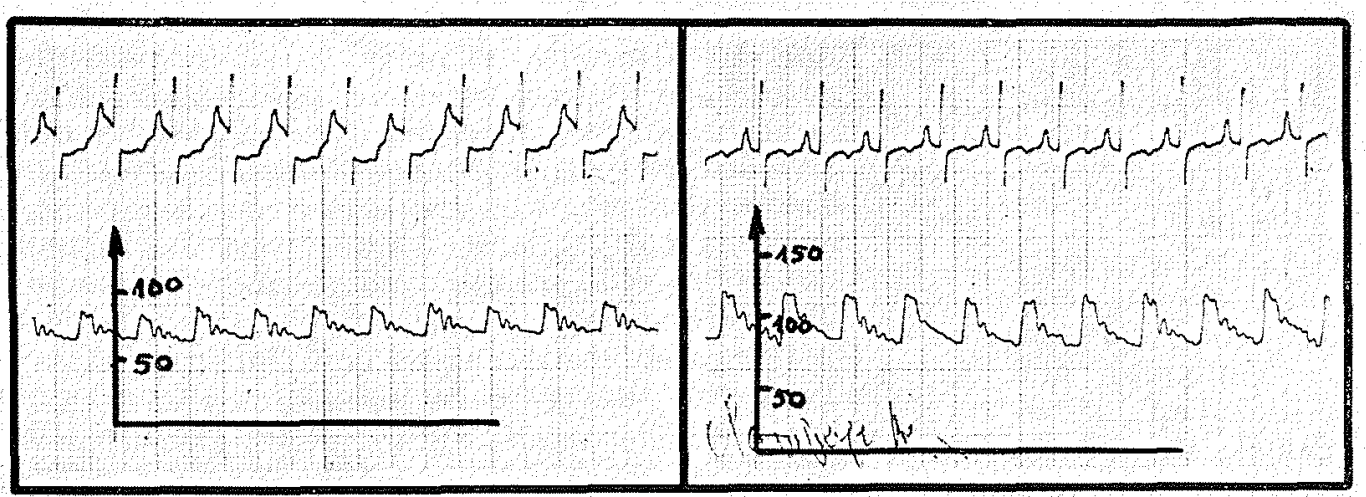

8/ Modification de l'EGG par' clampage de l'arte, suppression des signes d'ischémie. Expérience sul le chien.
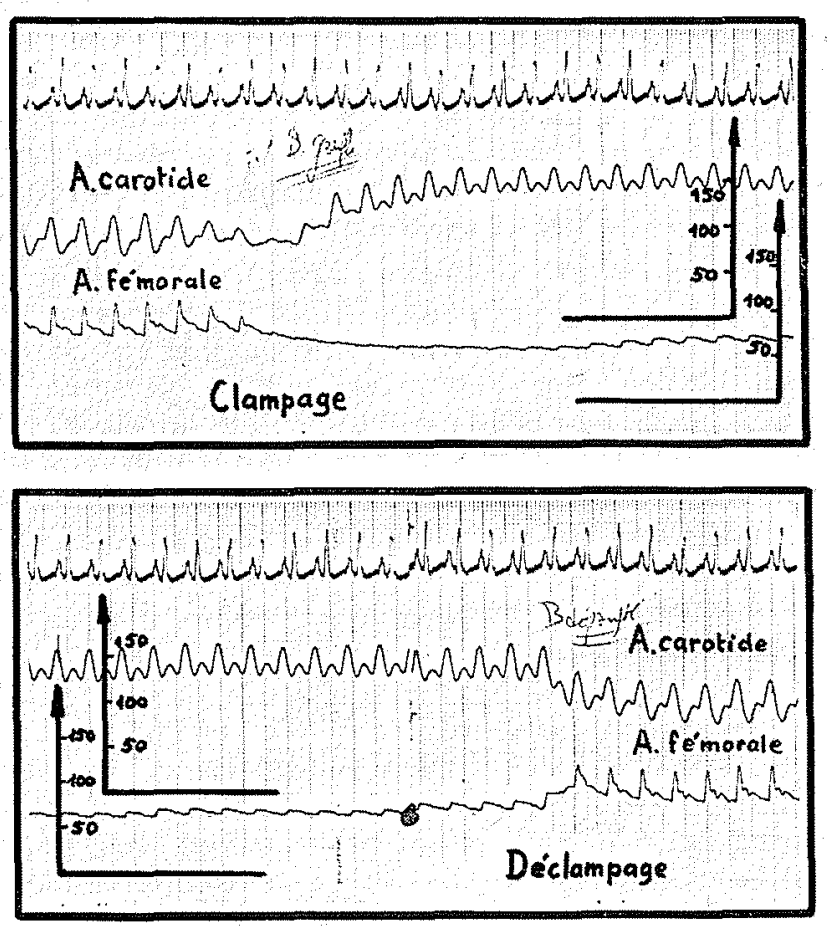

9/ Enregistrement de l'évolution des pressions au clampage partiel et au declampage de l'aolte. Fxpérience sur le chien.

lorsque le débit du shunt extérieur décroit traduit le fait que le débit d'irrigation supérieur augmente dans le même temps; le débit cardiaque restant à peu près constant, tout au moins au voisinage de la pression artérielle normale. Le débit du shunt extérieur continuant à diminuer, l'augmentation de pression supérieure entraîne une baisse du débit cardiaque, d'où l'aplatissement de la courbe.

Nous voilà certes assez loin des hypothèses de départ, puisque la réduction du débit inférieur entraine d'abord un accroissement d'énergie cardiaque. On ne voit ensuite l'énergie cardiaque diminuer que lorsque la courbe de pression supérieure s'infléchit. L'efficacité de la manœuvre réside pour la plus grande part dans l'accroissement de l'irrigation coronaire consécutive à l'augmentation de pression supérieure. Cette efficacité résultant d'un nouvel équilibre établi entre charge imposée au ventricule et apport par l'irrigation coronaire dépend essentiellement des valeurs de débit et pression pour lesquelles la courbe de pression supé-
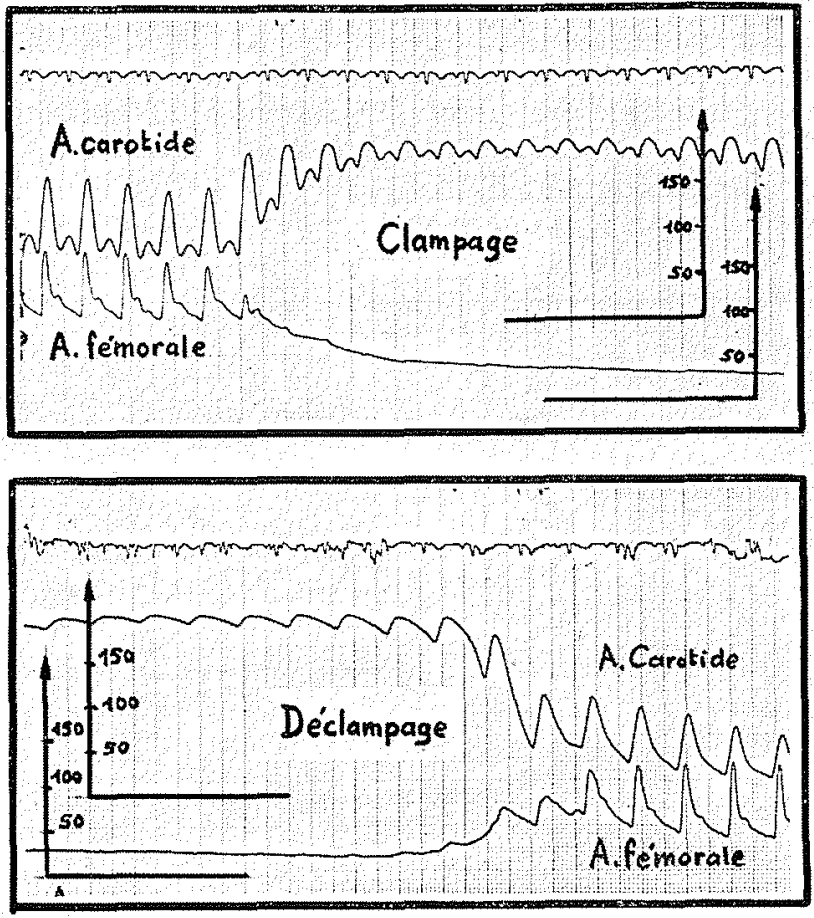

10/ Enregistrement de l'évolution des pressions au clampage total et au déclampage de l'aorte. Expérience sur le chien. rieure s'infléchit. On peut d'ailleurs considérer que la forme de cette courbe traduit la tonicité myocardique.

L'emploi du shunt extérieur avec pompe doseuse, commode du point de vue expérimental, n'est pas une nécessité. Beaucoup plus simplement, on peut par un gonflage partiel du ballonnet réaliser un clampage partiel de l'aorte. Dans ce cas, la simple connaissance des pressions permet de contrôler le débit d'irrigation inférieur. Le caractère pulsatile atténué de la pression inférieure est une caractéristique du clampage partiel dans ce cas, mais les considérations précédentes subsistent évidenment.

En résumé, l'application du principe du cloisonnement de la grande circulation par clampage de l'aorte, que ce soit avec ou sans le secours de l'hypothermie splanchnique, se révèle une manœuvre complexe dont l'efficacité est moins due à une réduction de débit cardiaque qu'à un accroissement du débit coronaire. Les expériences sur lesquelles 
nous nous sommes appuyés permettent certes de mieux comprendre le mécanisme de l'intervention, mais elles montrent aussi que le problème doit être examiné au niveau des échanges coronaires. En d'autres termes, l'amélioration du bilan cardiaque sera obtenu moins par une diminution de la dépense énergétique, que par un accroissement de l'apport métabolique. Il y a, à la réussite de l'opération, deux conditions : le coeur doit être tout d'abord capable de fournir temporairement le petit surcroit de pression qui lui permettra d'augmenter son débit coronaire, le tissu myocardique ensuite doit ètre capable de tirer profit de cet apport supplémentaire. L'isolement complet d'une partie de la grande circulation avec C.E.C. oxygénée a par contre l'avantage de réduire la dépense énergétique cardiaque, ce qui, joint à l'accroissement de l'apport métabolique, améliore les possibilités d'assistance.
M. Vador pose deux questions au Dr Hivglads :

$1^{\circ}$ Est-ce que l'on connaît le comportement de la pression ventriculaire gauche et la consommation d'oxygène pout un coule très ischémique ou un coeur très insuffisant;

$2^{\circ}$ Est-ce que l'on connait le comportement de cette courbe dans la zone située nettement à droite Autrement dit, que se passe-t-il si, au lieu d'abaisser cette pression ventriculaire, on laceroit? Ne va-t-on pas plafonner du point de vue consommation d'oxygène? A-t-on le droit d'extrapoler beaucoup cette courbe vers la droite? N'at-on pas une partie très descendante accompagnant la chute des pressions ventriculaires?

M. Hinglats rappelle que M. Mores arait dejà fort justement argumenté ce point et précise que les mesures portaient sur des myocardes sains et dans des conditions relativement physiologiques où les mécanismes d'auto-régulation du cour jouent encore bien.

M. Hinglars, par contre, ne pense pas que le gonflement du ballonnet dont a parlé M. VADOT puisse apporter un soulagement à un cour intrinsèquement défaillant. Lorsque l'on provoque un choc hémorragique, dans le premier temps de ce choc le coeur est sain; à $50 \mathrm{~mm}$ environ de pression de mercure, il survit assez tranquillement, tout en commencant à montrer, sur l'électrocardiogramme, des signes d'ischémie qui témoignent d'un assez mauvais remplissage de son lit coronaire : une heure ou une heure et demie après, se produisent al niveau du myocarde des lésions irréversibyles secondaires à ces ischémies. Il suffit de rétablir une pression artérielle nomale, e'est-à-dice supérieure à 60,80 ou $100 \mathrm{~mm}$ de mercure, pour restituer immédiatement une circulation coronaire convenable.

Gonfler un ballonnet artique et remonter la pression aortique doivent done immédiatement restituer au coeur sa bonne irrigation coronaire, si l'on n'a pas atteint le stade irréversible de lésions myocardiques dues au choc. Toutefois, cela entraine une surcharge et une augmentation considérable de la consommation myocardique; un ventricule véritablement défaillant, c'est-à-dire un ventricule dont la défaillance serait la cause de la chute de pression dans le lit artériel et non l'inverse, (c'est-à-dire une chute de pression antérielle cause d'une apparente ischémie myocardique) ne tolère pas une seconde une surcharge provoquée par un ballonnet intra-aortique.

D'autre part, l'hypothemie des telritoires sous-diaphragmatiques, que M. Vabot envisage précisément comme une méthode permettant, par la réduction du débit, de prolonger l'assistance circulatolre, paraît au contraire a M. HiNGLAIs soulever, par sa prolongation, des problèmes difficiles, tels que la mise en sommeil par réfrigération des fonctions hépatiques et rénales, si nécessaires pour corriger les altérations provoquées par la circulation extra-comporelle. Les expériences d'hypothermic profonde ont montré qũe tous les principes enzymatiques qui conditionnent ces fonctions se trouvent bloqués au-dessous de températures de 25 à $20^{\circ} \mathrm{C}$.

\section{Vador répond à M. Hinglais :}

10 Les tracés qu'il a projetés, en particulier le dernier, relatif à l'évolution des pressions, sont des tracés à caractère caricatural, en ce sens qu'il n'est pas obligatoire d'aller jusqu'à de pareilles surpressions et que celles-ci ne constituent donc pas un élément thérapeutique; au contraire, M. Vador pense les utiliser comme un moyen permanent d'assurer une réduction des débits, pour améliorer temporairement l'irrigation;

2 Par contre, en ce qui concerne l'hypothermie de l'aire sous-diaphragmatique, M. VADOT' est d'accord avec M. HiNGLAIs. Toutefois, il n'est pas dans les vues de M. LATREILLE et de M. VADOT de baisser les températures de l'aire sous-diaphragmatique a un niveau tel que les fonctions hépatiques et rénales soient quasi supprimées; ils désirent seulement analyser l'effet produit par l'hypothermie ou le clampage, afin de voir comment une combinaison possible de ces deux gestes pourait conduire à une réduction suffisante du débit cardiaque total permettant d'assurer une certaine assistance. Mais ils se gardent bien d'affirmer que la chose est possible. La meilleure preuve en est que le dernier procédé dont M. Latreille a parlé, celui de la circulation extra-corporelle banale, fait retomber sur le problème classique non encore bien résolu de l'oxygénation et pour lequel M. LATREILle propose l'emploi d'un poumon isolé.

Sur une question de M. BARgETon, M. VADOr indique qu'il n'a pas mesuré le débit coronaire, mais qu'il aurait pu le faire simplement par canulation du sinus coronaire.

M. BARgEToN remarque que, si on augmente la pression de perfusion, on augmente également la pression intra-ventriculaire et, par conséquent, aussi la résistance coronaire : dans ces conditions, il n'est pas certain que le gain que l'on pourrait espérer du fait de l'augmentation de la pression de perfusion ne soit pas diminué, on mème inversé, du fait de l'augmentation de résistance. C'est pourquoi il serait important d'avoir la possibilité de savoir comment évolue le débit coronaire

M. Vabot pense que, dans ces conditions, cette résistance serait uniquement systolique et que l'augmentation de pression dans la période diastolique resterait efficace.

Répondant à une question de M. Macarre, M. Monet indique que le cour peut très bien travailler même avec une mauraise oxygénation du sang arteriel et que les performances du cour ne dépendent pas uniquement de la quantité de sang arivant au cceur, de la pression artérielle ou de la saturation en oxygène: cela dépend également d'autres facteurs tels ce que l'on appelle l'inotropisme du myocarde : c'est ses propriétés contractiles, la fréquence cardiaque, les pressions intrathoraciques, les pressions intramurales, etc.

M. LaTrEILre ajoute une remarque en réponse à l'intervention de $M$. Hivglats : il ne faut pas considérer la méthode proposee comme une simple ocelusion de l'aorte, il faut prévoir automatiquement, et même avant l'occlusion, un court-circuit qui provoque wne décharge; le ballonnet ne peut etre gonflé que dans la mesure où le court-circuit fonctionne bien et peut-être faut-il d'abord faire le courtcircuit et ensuite gonfler le ballonnet.

M. Bonnis, a propos de la communication de MM. LATREILlE et VADot, voudrait compléter une réponse qui a été faite à une question posée sur les possibullités techniques de remédier, en général, à une pompe défectueuse et, en particulier, a une défectuosité d'une pompe circulatoire essentielle.

Dans l'industrie, on peut, en pareil cas recourir aux pièces de rechange et, lorsque l'organe à alimenter est vital, à la mise en parallele de deux pompes ou à la mise en réserve d'une pompe de secours.

En médecine, on ne peut pas avoir en réserve un organe 
identique au ccur et on utilise une pompe d'un autre type dont on modifie les caractéristiques en limitant sa puissance, sa pression ou probablement une donnée encore plus complexe, car on ne parait pas comnaitre encore la caractéristique hydraulique du cour, considéré comme une pompe. On utilise donc une pompe d'un type différent, généralement alternative, on la commande depuis l'extérieur et on l'installe soit en série, soit en parallèle, suivant les caractéristiques que l'on pourra relever.

La dernière communication montre une autre possibilité, que l'on utilise aussi d'ailleurs, dans l'industrie : c'est de réduire la puissance demandée en modifiant, non pas la caractéristique d'alimentation, mais celle du réseau récepteur. Mais la nécessité d'introduire un refrigérant, donc une perte de charge importante, oblige à avoir une deuxième pompe, placée loin de la première. Il semble, d'après certains schémas, que l'on peut utiliser une pompe à résime pratiquement continu à cause d'un amortissement très important des ondes de fonctionnement discontinu de pompe alternative, telle que le cœur, entre cette pompe qui est le cour et l'autre. Dans tous les autres cas, les pompes installées en assistance cireulatoire étaient nécessairement à débit pulsé et devaient avoir un certain synchronisme avec le cour, avec, éventuellement, le déphasage; d'où la complexité des phénomènes observés.

M. Bonnin mapelle enfin que les chirurgiens thoraciques tentent de modifier les caractéristiques statiques du réseau sur lequel débite la pompe. Or, les accidents circulatoires paraissent associés dans certains cas, sinon dus, à une modification par vieillissement des caractéristiques dynamiques, se traduisant par une perte d'élasticité, justiciable, en particulier, du bon amortissement des ondes de la pulsation du cœur : on obtient aussi une augmentation du diametre, donc du volume des artères permettant, peut-être, d'apporter un remède.

M. Bonnar rappelle alors une idée de M. Réménténas, laquelle, pour des raisons technologiques, n'a guère pu trouver d'application jusqu'icj dans l'industrie : elle consiste à profiter du volume aceru des artères principales pour y ajouter suivant la longueur, un corps doué d'une grande élasticité qui se comprimerait sous l'effet de la pulsation et gui, pent-être, répondrait, plus ou moins partiellement, au problème, si toutefois les artères peuvent le supporter.

Il $y$ aurait, dit en terminant $M$. Bonnis, un certain nombre de points à examiner au sujet des techniques expérimentales, mais ce serait trop long...

M. le Président remercie M. Bonnw et rappelle que, dans le procédé dont a parlé M. Bonnin, il est nécessaire que le tube auxiliaire disposé dans le conduit principal, en vue de modifier la célérité des ondes dans ce conduit, ait une élasticité beaucoup plus faible que ce dernier: par exemple, un tube en caoutchouc rempli d'air et placé à l'intérieur sur toute la longueur d'une conduite en acier réduit la célérité de 1000 à $100 \mathrm{~m} / \mathrm{s}$, et au-dessous. Or, comme l'artère a tune célérité propre très faible, on ne voit pas quel genre de tuyau il faudrait placer daus lartère pour diminuer encore la célérite. On pourrat, théoriquement, augmenter l'efficacité du système en emplissant le tube d'une vapeur, eondensable par une très faible augmentation de pression, mais cela serait ici, bien compliqué.

M. Hiwglas indique qu'un procédé analogue a été réalisé par Moulopoulos : il introduisait dans le lit aorticque un ballonnet gonfle rythmiquement d'une centaine de millilitres d'air comprimé; ce ballonnet provoquait des à-coups de pression au moment de la diastole. Les résultats étaient jntéressants, mais l'apport énergétique réalisé par' ce système n'etait pas suffisant pour que ces expérimentations se soient poursuivies.

M. VADot précise que l'expérience de Moulopoulos est, en fait, une contre-pulsation : le contre-pulseur est placé à l'intérieur de l'aorte alors que l'idée de M. Réménnéras est une modification de la caracteristique de l'arte par l'introduction d'un tube rempli d'air et qui serait placé, par exemple, suivant l'axe de l'aorte; ce tube serait, du reste, statique et subirait des oscillations systoliques, mais son rôle se bornerait á modifier les caractéristiques d'élasticité et de vitesse d'onde de l'arte.

M. le Président rappelle que les divers systèmes de pompage mis en ouvre dans les assistances circulatoires donnent des variations de pression assez abruptes. On pourrait, très facilement, adoucir ces variations en introduisant des élasticités supplémentaires dans les tuyaux qui relient au réseau corporel la pompe placée à l'extérieur du corps : au lieu d'une section droite circulaire on pourrait envisager, pour la connexion en caoutchouc, une section oblongue ou en etoile, dont la variation du volume en fonction de la pression $d v / d p$ serait plus grande.

M. le Président remercie les conférenciers et les participants à la discussion. Il conclut en observant qu'en médecine, comme en hydraulique, seule l'expérimentation est capable d'apporter un supplement d'information; mais les mathematiques permettent souvent d'analyser celle-ci en vue d'une interprétation plus fine ou d'une éventuelle extrapolation. 